\title{
Corynebacterium simulans
}

National Cancer Institute

\section{Source}

National Cancer Institute. Corynebacterium simulans. NCI Thesaurus. Code 122275.

A species of nonlipophilic, non-motile, fermentative, catalase-positive bacteria in the family Corynebacteriaceae. C. simulans is positive for nitrate and the nitrite reductase test, does not ferment maltose, and is unable to acidify ethylene glycol. 